\title{
Geometric Sensitivity Index for the GNSS Using Inner Products of Line of Sight Vectors
}

\author{
Dae Hee Won* \\ University of Colorado, Boulder, Colorado 80309, USA
}

Jongsun Ahn** and Sangkyung Sung***

Konkuk University, Seoul 05029, Korea

\section{Chulsoo Lee ${ }^{* * * *}$ and Sungchun Bu ${ }^{* * * * *}$}

LIG Nex1 Co., Ltd, Seongnam 13488, Korea

\author{
Jeagyu Jang****** \\ Agency for Defense Development, Daejeon 34060, Korea
}

Young Jae Lee Le****** $^{*}$

Konkuk University, Seoul 05029, Korea

\begin{abstract}
Satellite selection and exclusion techniques have been applied to the global navigation satellite system (GNSS) with the aim of achieving a balance between navigational performance and computational efficiency. Conventional approaches to satellite selection based on the best dilution of precision (DOP) are excessively computational and complicated. This paper proposes a new method that applies a geometric sensitivity index of individual GNSS satellites. The sensitivity index is derived using the inner product of the line of sight (LOS) vector of each satellite. First, the LOS vector is computed, which accounts for the geometry between the satellite and user positions. Second, the inner product of each pair of LOS vectors is calculated which indicates the proximities of the satellites to one another. The proximity can be determined according to the sensitivity of each satellite. A post-processing test was conducted to verify the reliability of the proposed method. The proposed index and the results of a conventional approach that measures the dilution of precision (DOP) were compared. The test results demonstrate that the proposed index produces results that are within $96 \%$ of those of the conventional approach and reduces the computational burden. This index can be utilized to estimate the sensitivity of individual satellites, obtaining a navigation solution. Therefore, the proposed index applies to satellite selection and exclusion as well as to the sensitivity analyses of multiple GNSS applications.
\end{abstract}

Key words: GNSS, Sensitivity, Line of Sight Vector, Inner Product, Performance Index

\section{Introduction}

The global navigation satellite system (GNSS) has made significant progress in navigation applications, such as
This is an Open Access article distributed under the terms of the Creative Commons Attribution Non-Commercial License (http://creativecommons.org/licenses/by$\mathrm{nc} / 3.0 /$ which permits unrestricted non-commercial use, distribution, and reproduction in any medium, provided the original work is properly cited. in military, transportation, communication, and aviation navigation. Currently, several countries operate the GNSS or have developed their own GNSS because of its extensive effect on the industry as well as society, making the GNSS a

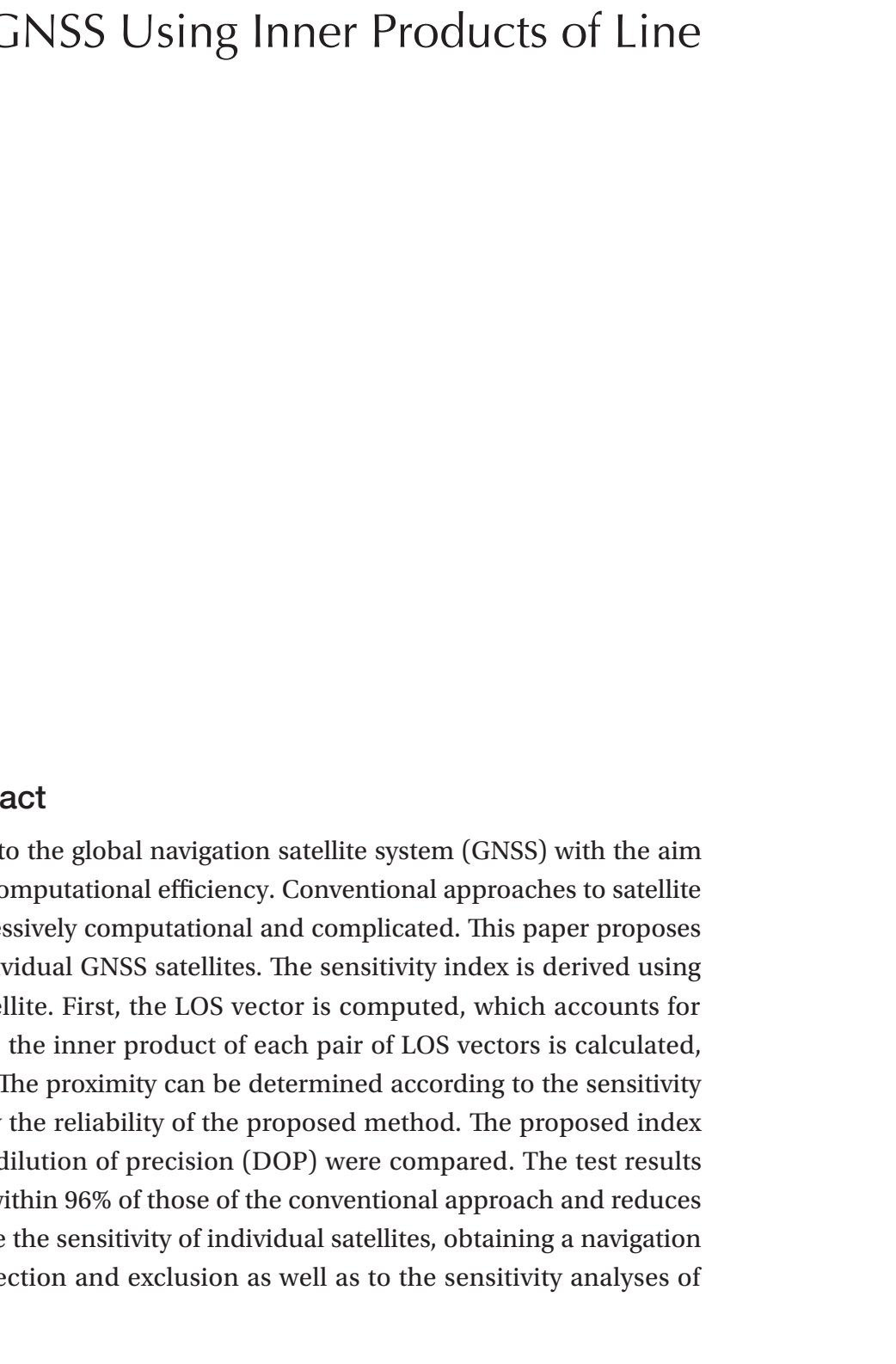

(c)

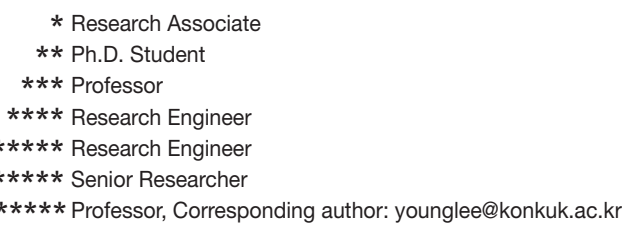

http://ijass.org pISSN: 2093-274x elSSN: 2093-2480 
key world infrastructure. Several GNSSs exist, such as the global positioning system (GPS) used in the United States, the global navigation satellite system (GLONASS) in Russia, Beidou in China, Galileo in the European Union, and the quasi-zenith satellite system (QZSS) in Japan [1, 2]. General users can utilize multiple systems due to their open access policy.

GNSSs consist of medium earth orbit (MEO) constellations, and some follow geostationary orbits (GEOs). These GNSS satellites continuously follow their own orbit and are not fixed at a certain point $[1,3,4]$. As a result, the satellite distribution in the sky is constantly changing simultaneously and periodically. Because the number of GNSS satellites is increasing, a substantial number of GNSS signals are available [2]. If the number of satellites increases, their distribution is more likely to be evenly dispersed and dense.

Satellite distribution, as well as the number of satellites, determines the navigational performance of the system. Although a user's navigational performance improves as the number of satellites increases, this relationship is not exactly proportional. Performance improvement increases at a slower rate after the number of satellites reaches in certain level because a large number of satellites will increase the computational complexity rather than the performance $[2,5]$. Therefore, determining the number of satellites that would optimize the user's overall navigational performance would be preferable to simply adding more satellites. For example, the exclusion of some of the visual satellites would be expected to effectively address navigational computing issues (position, velocity, or time) that arise with the use of a large number of satellites. In addition, the given satellite distributions may not be even and may include relatively dense or sparse areas; thus, excluding a few satellites from the dense areas will not diminish performance but could decrease the computational burden. However, the exclusion of satellites requires that two decisions be made: determining which satellites to exclude (or select) and the criteria used in this determination. A rule must be established in order to maintain a balance between the overall performance and computational efficiency. In this paper, we propose a simple sensitivity index.

During the initial stage of GPS receiver development, the available computational capacity could not process all the signals from every visual satellite [6]. Therefore, the need to reduce the computational burden gave rise to a demand to select a subset of visual satellites. Given the lowcost receivers, which had insufficient real-time processing capacities and a limited number of hardware channels, an appropriate satellite subset that would simultaneously reduce the computational burden and minimize performance degradation was needed. As time passed, electrical technology significantly developed, and the computational requirement was no longer problematic. The simultaneous processing of all visual satellite information became possible. However, other issues appeared, such as the cost of high-performance processors, power consumption, and computational efficiency. Furthermore, when the number of satellites reaches a certain level, the improvement of the navigational performance degrades or becomes saturated [2]. In this case, computational efficiency decreases as the number of satellites increases. In terms of overall efficiency, determining the optimal number of satellites, as well as the best subset of visual satellites, is preferable [2, 6-8].

In the early stages of GPS satellite selection, dilution of precision (DOP) was used as the selection policy. In [6, 9], vertical DOP (VDOP), horizontal DOP (HDOP), and geometric DOP (GDOP) were the criteria used to select satellites in order to obtain accurate navigational solutions. Although this approach presents the optimal criteria because it determines the subset that best minimizes the DOP, when considering all visible GNSS satellites, it needs to examine a substantial number of subsets. For example, in order to select 10 satellites from 20 satellites, 184,756 subsets $\left.{ }_{20} C_{10}=184,756\right)$ must be examined. As a result, this method is restricted or impossible to apply to real-time processing. In order to overcome this computational burden, several methods have been proposed. Zheng [10] presented a tetrahedron method, which maximizes the geometric volume using four satellites. It selects the three satellites that have the greatest separation angles in the north, east, and zenith directions and then selects one more from the remaining satellites. This method is effective and requires only four satellites. However, the computational problem still exists because all visible satellites must be examined. Zhang $[8,11]$ selected the satellite that had the greatest elevation angle. Subsequently, satellite selection was based on a criterion, wherein the satellites possessing the most perpendicular line of sight vector were selected first among the remainder satellites. Yan [12] proposed the selection of a subset of satellites based on a predefined database, which included sensitivity information about potential satellites. In GNSS real-time kinematic (RTK) applications, satellite selection has also been researched. These studies focus on finding one primary satellite by considering the DOP, observation time, and elevation angle [13, 14]. However, this approach is only effective in RTK applications because the subsets of satellites align with the primary satellite.

Previous approaches are too computationally demanding and time consuming. They require substantial computational 
resources as well as costly computing processors, and they are inefficient in real-time processing when determining the appropriate subsets of visible satellites. In this paper, we propose a geometric sensitivity index that is less computationally demanding yet applicable to satellite selection and exclusion. The index is derived using the inner product of the line of sight (LOS) vector of each satellite to determine the proximities between satellites. The LOS vector is computed by taking into account the geometry between the satellite and the position of the user. Individual LOS vectors are used to calculate the inner product of the vector of the other satellites, and then each satellite is assigned a score (index) by averaging these results. This score signifies the average proximity of a satellite to other satellites. The score is a distinct index and denotes the effectiveness of the satellite as a navigation solution. An experimental test was conducted in order to verify the reliability of this index. The proposed index was compared with the results obtained using the conventional approach, which determined subsets using the best DOP. Furthermore, a statistical analysis was conducted to find the most sensitive satellite, which could be used as the navigational solution. The test results indicate that the proposed index was within $96 \%$ of the results of the conventional approach and that it had a significantly lower computational burden. This index indicates the sensitivity of each satellite as a navigation solution. Consequently, the index can be applied to satellite selection and exclusion methods.

\section{GNSS Observation}

Before deriving the sensitivity index, GNSS observation should first be defined. A GNSS range measurement can be calculated using the propagation time between the satellite and the receiver. This measurement includes the geometric relationship between the satellite and user positions and the clock bias. In general, satellite clock bias can be corrected by broadcasting navigation messages, which allows us to assume that only the receiver clock bias term exists. Thus, the GNSS observation equation is given by $[1,3]$

$$
\rho_{u}^{s}=\sqrt{\left(x^{s}-x_{u}\right)^{2}+\left(y^{s}-y_{u}\right)^{2}+\left(z^{s}-z_{u}\right)^{2}}+c \Delta b_{u}
$$

where

$$
\begin{aligned}
& \rho_{u}^{s}: \text { measured pseudorange } \\
& {\left[\begin{array}{lll}
x^{s} & y^{s} & z^{s}
\end{array}\right]: \text { satellite position in } \mathrm{x}-\mathrm{y}-\mathrm{Z} \text { axis }} \\
& {\left[\begin{array}{lll}
x_{u} & y_{u} & z_{u}
\end{array}\right] \text { : user position in } \mathrm{x}-\mathrm{y}-\mathrm{Z} \text { axis }} \\
& c: \text { speed of light } \\
& \Delta b_{u} \text { : clock bias of receiver }
\end{aligned}
$$

Equation (2) and (3) are obtained by linearizing equation (1) with respect to nominal user position $\mathbf{x}_{u 0}$, expanding this for all visible satellites.

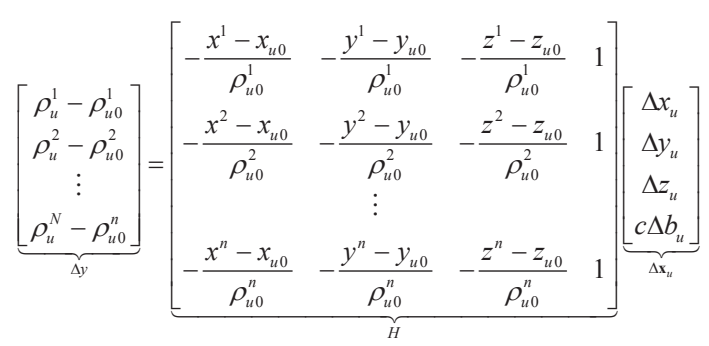

$\Delta y=H \Delta \mathbf{x}_{u}$

Here,

$\mathbf{x}_{u 0}=\left[\begin{array}{llll}x_{u 0} & y_{u 0} & z_{u 0} & c \Delta b_{u 0}\end{array}\right]^{T}$

$\rho_{u 0}^{s}=\sqrt{\left(x^{s}-x_{u 0}\right)^{2}+\left(y^{s}-y_{u 0}\right)^{2}+\left(z^{s}-z_{u 0}\right)^{2}}+c \Delta b_{u 0}$.

$n$ : total number of visible satellites

Observation matrix $H$ in equation (3) consists of LOS vectors and a clock bias term. Estimating the position corrections using the least-squares method allows the user position to be estimated by compensating nominal point $\mathbf{x}_{u 0}$ in equation (4).

$$
\Delta \hat{\mathbf{x}}_{u}=\left(H^{T} H\right)^{-1} H^{T} \Delta y
$$

\section{Optimal Performance Index}

The DOP is a multiplicative parameter that converts the pseudo-range measurement error into the coordinates of the user position for a given satellite distribution. In general, the DOP has been used in satellite constellation design, performance evaluation, and the prediction of expected navigational error levels. The DOP used in this method is derived from the error covariance, which is calculated from the least-squares estimation equation $[4,15]$. Applying the general definition of the DOP gives us equations (5) and (6). Details of the DOP follow.

$$
D=\left(H^{T} H\right)^{-1}
$$

$$
\text { DOP }= \begin{cases}\text { Geometric DOP }(\text { GDOP })= & \sqrt{D_{11}+D_{22}+D_{33}+D_{44}} \\ \text { Position DOP }(P D O P) & =\sqrt{D_{11}+D_{22}+D_{33}} \\ \text { Horizontal DOP }(H D O P) & =\sqrt{D_{11}+D_{22}} \\ \text { Vertical DOP }(\text { VDOP }) & =\sqrt{D_{33}} \\ \text { Time DOP }(T D O P) & =\sqrt{D_{44}}\end{cases}
$$

Here, $D_{i i}$ refers to the $i^{\text {th }}$ diagonal element (i.e., the element 
in the $i^{\text {th }}$ row and $i^{\text {th }}$ column) of matrix $D$.

The DOP has also been historically used as a performance index in GNSS applications. It is an optimal criterion for both satellite selection and performance evaluation. In order to achieve an optimal satellite subset selection, it selects the satellite subset having the minimal DOP after computing all the DOPs of possible satellite subsets.

$$
\text { Optimal selection: } \min \left[\operatorname{DOP}\left({ }_{n} C_{m}\right)\right]
$$

In this equation, $n$ is the total number of visible satellites, $m$ is the number of selected satellites, and $C$ is the combination process. This method guarantees the best satellite selection, but it requires a substantial amount of computation. When considering all visible GNSS satellites, a significant number of subsets must be examined. For example, if ten satellites are selected from twenty satellites, 184,756 subsets $\left({ }_{20} C_{10}=184,756\right)$ must be examined. Moreover, the matrix inversion in equation (5), which is computationally expensive, should be repeated for every subset in the process. As a result, this method is limited and may not apply to realtime processing.

Other conventional approaches [5-13] are only valid for satellite selection and exclusion and do not provide a sensitivity index for individual satellites. Therefore, approaches other than the optimal index (DOP-based) method should not be compared with the index proposed in this paper.

\section{Proposed Sensitivity Index}

Each satellite has its own elevation and azimuth angle as well as its position within the geometry, and these aspects affect the navigational solution. In other words, each satellite has a different sensitivity to computing the position solution. Therefore, the sensitivity of each satellite should be calculated.

The proposed approach applies the inner product of the LOS in order to determine the proximity of the LOSs to one another. The inner product of two vectors calculates the angle between the vectors, determining their independence. The inner product of two unit vectors $(\vec{a}, \vec{b})$ lies in the range between -1 and 1 . If the vectors are nearly equivalent, then the inner product is close to 1 , whereas an inner product of 0 indicates that the two vectors are perpendicular. Two opposite vectors have an inner product of -1 . Therefore, the proximity between two vectors can be determined using the inner product in equation (8).

$$
\begin{aligned}
\vec{a} \cdot \vec{b} & =|a||b| \cos \theta \\
& =\cos \theta
\end{aligned}
$$

We can extend this inner product concept to satellite LOSs. Observation matrix $H$ in equation (3) contains sufficient information to compute the inner product for all visible satellites. Each satellite has its own LOS, which is represented in the first three columns, denoting the $x-y-z$ direction from the user to the satellite. In equation (9), LOS components are presented as $u_{x}, u_{y}, u_{z}$ for each direction and $\mathrm{n}$ denotes the number of satellites. Moreover, calculating the inner product is simple because all vectors are unit vectors, and it does not require considering the size of each LOS vector in equation (8). The angle in cosine form can be directly computed from the inner product without compensating for vector size.

If we assume the LOS vector excludes the clock term, then the modified observation matrix can be obtained. This enables us to check the effect of geometrical sensitivity only, i.e., without using the clock term, as the optimal approach only uses PDOP rather than GDOP with the clock term.

$$
\begin{aligned}
\tilde{H} & =\left[\begin{array}{ccc}
u_{1, x} & u_{1, y} & u_{1, z} \\
u_{2, x} & u_{2, y} & u_{2, z} \\
& \vdots & \\
u_{n, x} & u_{n, y} & u_{n, z}
\end{array}\right]=\left[\begin{array}{c}
\mathbf{u}_{1} \\
\mathbf{u}_{2} \\
\vdots \\
\mathbf{u}_{n}
\end{array}\right] \\
P & =\tilde{H} \tilde{H}^{T} \\
& =\left[\begin{array}{cccc}
1 & \cos \theta_{12} & \cdots & \cos \theta_{1 n} \\
\cos \theta_{21} & 1 & \cdots & \cos \theta_{2 n} \\
\vdots & \vdots & \ddots & \vdots \\
\cos \theta_{n 1} & \cos \theta_{n 2} & \cdots & 1
\end{array}\right]
\end{aligned}
$$

The modified observation matrix contains all LOS vectors, and all possible inner products $(P)$ can be simultaneously computed by multiplying $\tilde{H}$ and its transposed matrix $\tilde{H}^{T}$ . The result should be a square matrix, and its dimension equals the number of visible satellites. The element in the $i^{\text {th }}$ row and $j^{\text {th }}$ column denotes the inner product of the $i^{\text {th }}$ and $j^{\text {th }}$ satellites, and it is easily transformed into the cosine of the angle $\left(\theta_{i j}\right)$ induced between the two LOS vectors. Therefore, $P$ includes information that is useful in analyzing the closeness of all visible satellites.

Matrix $P$ has two distinct characteristics. First, it is symmetric, which implies that all of its components do not need to be analyzed. In other words, only the upper (or lower) triangle matrix is required to determine the closeness of all visible satellites. Second, all diagonal terms are 1 because they are inner products of themselves (if $i=j$, then $\cos \theta_{i j}=1$ ) and can be excluded from the closeness analysis.

Analyzing matrix $P$ enables us to find the closest satellite by searching the maximum component, which is determined by two satellites. The inner product returns a scalar value from two vectors. Thus, the maximum inner product is the product of two satellites. Checking only the maximum inner 
product is insufficient, and the search must be extended to the second greatest inner product or possibly further. Then the index can include the proximity with several neighbor satellites. Subsequently, these inner products are averaged, and this average signifies the score value (index) for each satellite. The proposed sensitivity index (S) can be expressed as

$$
S(i)=E\left[\max _{2}\left(P_{i, \text { all }}\right)+\max _{3}\left(P_{i, \text { all }}\right)+\cdots+\max _{n}\left(P_{i, \text { all }}\right)\right]
$$

where $\max _{n}$ is the $n^{\text {th }}$ maximum value, $P_{i, \text { all }}$ represents the vector in the $i^{\text {th }}$ row and every column, and $i$ denotes the satellite index. In equation (11), the first maximum is not used because it is computed alone and always produces a value of 1 .

\section{Tests and Analysis}

The proposed sensitivity index was verified through an analysis of experimental data. The results were compared with the conventional optimal index approach (in section 3) in terms of computational time and the optimality of satellite selection.

\subsection{Test Settings}

The test data were generated using the version 3.0 receiver independent exchange (RINEX3) navigation data from the National Aeronautics and Space Administration (NASA) Crustal Dynamics Data Information System, (CDDIS) website (http://cddis.nasa.gov/). The data were referenced on December 24, 2014, and included multiple GNSS constellations (GPS, GLONASS, Gaileo, Beidou, and QZSS) over a 24-hour period. The receiver antenna was assumed
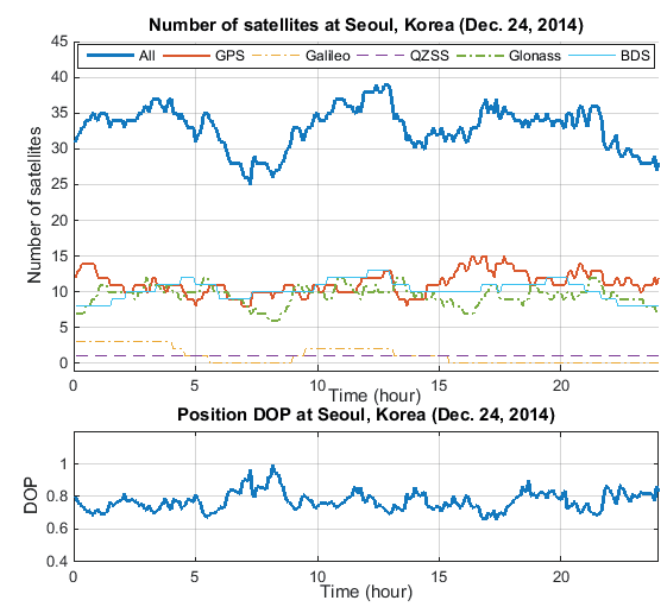

Fig. 1. Number of visual satellites (top) and DOP (bottom) over 24 hours to be located in Konkuk University, Seoul, Korea, because Korea has a geographical advantage when receiving various GNSS signals.

The data was post-processed using ®Matlab 2014b on Windows 7 (i7 processor, 8 GB RAM). The number of satellites ranged from 25 to 39, and the average was 33 for the 24 -hour period. Fig. 1 displays the number of satellites and the PDOP over time, and the satellite distribution can be seen in Fig. 2 . The satellite cut off angle was 0 deg during the test.

The performance of the proposed index was verified by analyzing its performance in comparison to the optimal satellite selection achieved by using the DOP. The satellite selection achieved using the DOP is always optimal and is henceforth referred to as the optimal index. Therefore, comparing the optimal index with the proposed sensitivity index (S) is a reasonable method to use in the performance evaluation. The proposed sensitivity index was computed using equation (11), whereas the optimal index was obtained by computing the DOP of every possible subset of visible satellites. As the range of the two indexes differed, a normalization process was applied to adjust the scale. The normalization was achieved by dividing a distance from the mean (value minus mean) by the standard deviation.

\subsection{Test Results}

The proposed sensitivity index was verified by analyzing the result that was obtained by determining the optimal index. The computation time and sensitivity accuracy were examined.

First, the computation time was analyzed. The number of satellites to be selected varied, and the resulting computation time was measured. Table 1 exhibits the computation times over 24 hours using 300-second intervals, and the number of satellites (\# Satellites) is incremented from one to five. Here, the number of satellites indicates the number of satellites to be selected or excluded. For example, the satellite selection

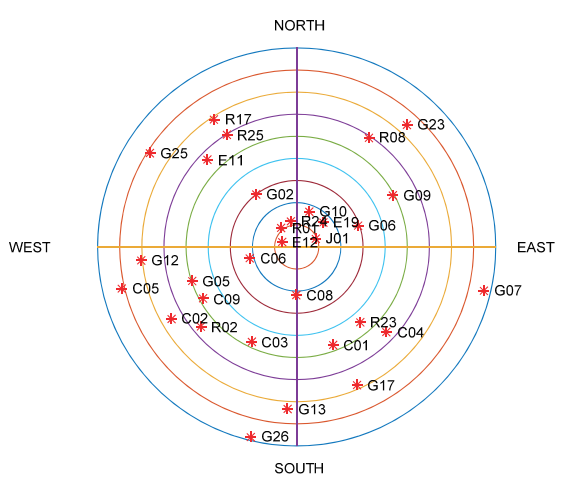

Fig. 2. Skyplot at 2:00 on December 24, 2014: G, J, R, C, and E represent GPS, QZSS, GLONASS, Beidou, and Galileo, respectively 
process required two steps in order to select two satellites because the index produced in the first step was valid only for a given visible satellite set. Therefore, the indexes were computed again after the visible satellite set changed according to the satellite selections or exclusions. As the number of satellites increased, the computation time for the proposed index also increased linearly. However, the optimal index increased exponentially. Given one satellite selection, the optimal index was approximately 20 times as computationally expensive as the proposed one.

The optimal index should check all possible subsets of given visible satellites, as in equation (5).

Figure 3 shows the number of subsets that test the DOP in order to form the optimal index. Each line denotes the total number of visible satellites, which ranged between 20 and 70 (assuming full GNSS constellation and $50 \%$ visibility). The $x$-axis represents the number of satellites to be selected, and the $y$-axis measures the number of subsets to be tested. The number of subsets increased until it reached half of the entire number of given satellites because the process for selecting satellites is equivalent to the process for excluding them. This increasing number of subsets results in a substantial computational burden. Moreover, the DOP computations should be performed in every subset, and the computation points equal the number of satellites within a given subset. Therefore, the proposed sensitivity index is more efficient in terms of processing time, whereas the optimal index is computationally expensive.

Second, the accuracy of the proposed sensitivity index was verified by checking its correspondence with the optimal index. Fig. 4 illustrates the distribution of visible satellites after seven hours. In this case, 26 satellites were visible. Fig. 5 displays the normalized optimal and proposed sensitivity index for each individual satellite represented in Fig. 4. The optimal index was obtained by computing the DOP of the satellite subsets, which successively subtracted each satellite, and determining an inverse because the DOP demonstrated an inverse relationship with the proposed sensitivity index. The proposed sensitivity index was computed using equation (11). Both indexes were normalized in order to create a more accurate analysis that accounted for scale. The $x$-axis denotes the pseudo-random number (PRN), and the $y$-axis represents the normalized index. The proposed index in the top graph used equation (11) to average all the satellites, and the proposed index in the bottom graph averaged only the first two maximum values. Lesser values signify greater sensitivity, and greater values imply less sensitivity. The optimal and proposed sensitivity index trends were approximately similar, and the correlation coefficients between the two indexes were 0.781 (top) and 0.852 (bottom). The proposed index adequately followed the optimal index in the high-sensitivity region (below -1), although it demonstrated some inconsistencies in the low-sensitivity region (around 0).

Table 1. Computation time (seconds) with respect to the iterations necessary to check the indexes

\begin{tabular}{cccc}
\hline \# Satellites & $\begin{array}{c}\text { Optimal index } \\
(\mathrm{sec})\end{array}$ & $\begin{array}{c}\text { Proposed index } \\
(\mathrm{sec})\end{array}$ & $\begin{array}{c}\text { Ratio } \\
(\%, \text { optimal/proposed })\end{array}$ \\
\hline 1 & 0.461 & 0.022 & 20.955 \\
2 & 3.169 & 0.047 & 67.426 \\
3 & 46.332 & 0.065 & 712.800 \\
4 & 391.727 & 0.078 & 5022.141 \\
5 & 2158.032 & 0.093 & 23204.645 \\
\hline
\end{tabular}

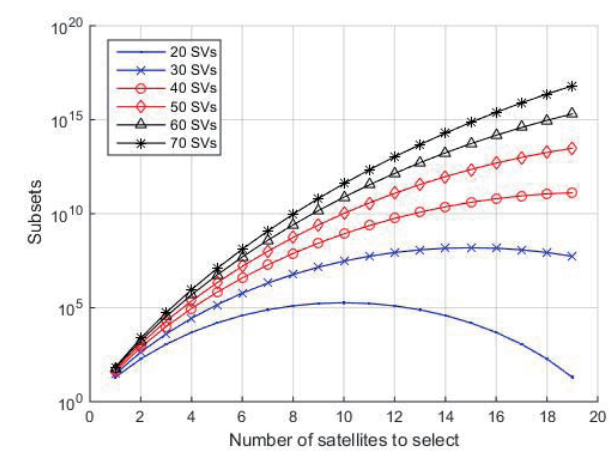

Fig. 3. Number of subsets on which the DOP was tested in the optimal index case with respect to the total number of visible satellites

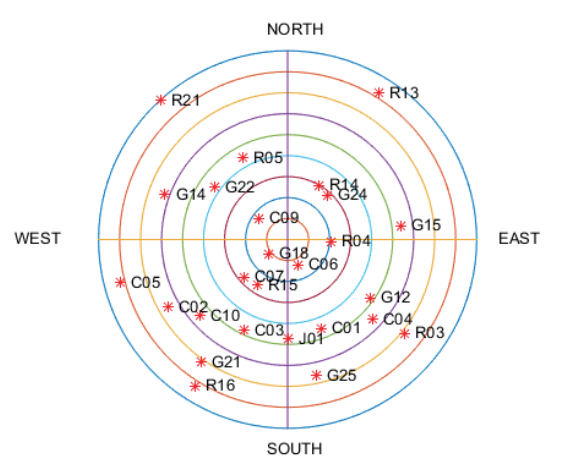

Fig. 4. Skyplot after seven hours 
Figure 6 shows the test results to determine the extent to which the sensitivity of the proposed and optimal methods corresponds. This index is produced by comparing the order of sensitivity. Once the sensitivity indexes are computed using both the optimal and proposed methods, each index is sorted by its order of sensitivity. After the index is sorted, the most sensitive satellite is determined using the proposed sensitivity index, and its position is determined using the optimal index. If the most sensitive satellite derived from the proposed method also ranks highest in the optimal index list, then the test result receives a 1 . If it ranks third in the optimal index list, then the correspondence index is $(n-3) / n$, in which $n$ is the total number of visible satellites. The test results demonstrate that the two indexes coincided $95.9 \%$ of the time when selecting the most sensitive satellite.

The proposed sensitivity index and the optimal index exhibit similar trends. Increasing the accuracy of the proposed index requires the elevation angle of the satellites to be considered. Most GNSS applications use the satellites that are above the horizon (have a positive elevation angle) because of their visibility. The greatest possible elevation angle is 90 degrees; thus, the maximum angle induced with the highest satellite is less than 90 degrees. This implies that the highest satellite will more likely demonstrate positive inner products when compared with other satellites. If the average is taken over all the inner product components of a given satellite, the highest satellite is at a disadvantage.
However, because higher satellites produce more effective navigation solutions [15] a compensation (or weighting) process must be applied in addition to the proposed approach to improve the accuracy.

\section{Conclusion}

Satellite distribution determines the navigational performance, and the number of satellites is proportional to the navigational performance. However, a significant number of satellites increase the computational burden rather than increasing the performance. Therefore, the selection of an appropriate number of satellites improves the overall performance of the user's navigational system, and a rule that balances the overall performance improvement and computational efficiency must be established. In this paper, we propose a geometric sensitivity index, which is simple and fast as well as less computationally intensive, for satellite selection and exclusion.

The index is derived using the inner product of the LOS vector of each satellite to determine the proximity of a satellite to its neighbors. Individual LOS vectors are used to determine these inner products, and then each satellite is assigned its index by averaging the results. This indicates the average proximity of a satellite to other satellites. An experimental test was conducted to verify the reliability
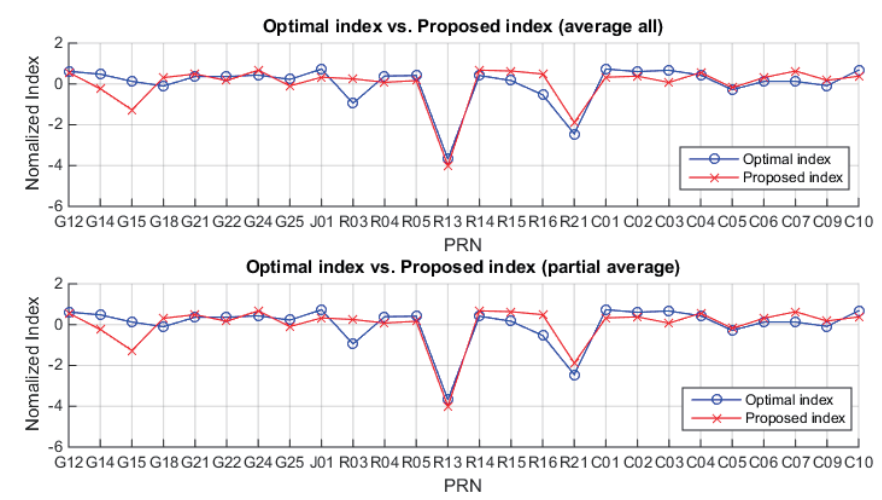

Fig. 5. Normalized performance index for the optimal index and proposed index, computed by averaging all (top) and the first two (bottom) maximum values

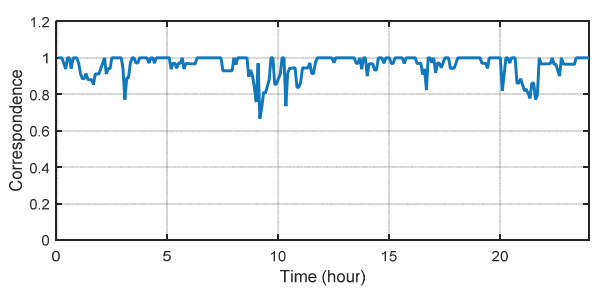

Fig. 6. Correspondence between the two indexes over time. of this index, which was analyzed by comparing it with the index produced by a conventional approach that tests subsets according to the best DOP. Furthermore, a statistical analysis was conducted to determine the most sensitive satellite for the navigation solution. The test results reveal that the proposed sensitivity index is close to the index determined by the conventional approach $96 \%$ of the time and exhibits a reduced computational burden. This index 
indicates the effectiveness of each satellite as a navigational solution. This implies that the proposed index can be used for satellite selection or exclusion and sensitivity checks.

\section{References}

[1] B. Hofmann-Wellenhof, H. Lichtenegger, and E. Wasle, GNSS-global navigation satellite systems : GPS, GLONASS, Galileo, and more. Wien ; New York: Springer, 2008.

[2] G. Bibbons, "GNSS Interoperability: Not So Easy, After all”, Volume 6, Number 1 January/February, ed: InsideGNSS, 2011, pp. 28-31.

[3] E. D. Kaplan, and C. Hegarty, Understanding GPS : principles and applications, 2nd ed. Boston: Artech House, 2006.

[4] B. W. Parkinson, and J. J. Spilker, The global positioning system : theory and applications. Washington, DC: American Institute of Aeronautics and Astronautics, 1996.

[5] M. Liu, M. A. Fortin, and R. Landry, "A Recursive Quasioptimal Fast Satellite Selection Method for GNSS Receivers", Proceedings of the 22nd International Technical Meeting of the Satellite Division of the Institute of Navigation (Ion Gnss 2009), 2009, pp. 2061-2071.

[6] M. Kihara, "Study of a GPS Satellite Selection Policy to Improve Positioning Accuracy", IEEE 1994 Position Location and Navigation Symposium, 1994, pp. 267-273.

[7] M. R. Mosavi, and M. Sorkhi, "An Efficient Method for Optimum Selection of GPS Satellites Set using Recurrent Neural Network", 2009 Ieee/Asme International Conference on Advanced Intelligent Mechatronics, Vol. 1-3, 2009, pp. 245-249.
[8] M. Y. Zhang, and J. Zhang, "A Fast Satellite Selection Algorithm: Beyond Four Satellites", Ieee Journal of Selected Topics in Signal Processing, Vol. 3, Oct 2009, pp. 740-747.

[9] M. S. Phatak, "Recursive method for optimum GPS satellite selection", Ieee Transactions on Aerospace and Electronic Systems, Vol. 37, Apr 2001, pp. 751-754.

[10] Z. Y. Zheng, C. Huang, C. G. Feng, and F. P. Zhang, "Selection of GPS satellites for the optimum geometry", Chinese Astronomy and Astrophysics, Vol. 28, Jan-Mar 2004, pp. 80-87.

[11] M. Y. Zhang, J. Zhang, and Y. Qin, "A new quickly algorithm for GPS/Galileo/BD satellite selection", Second International Conference on Space Information Technology, Pts 1-3, Vol. 6795, 2007, pp. I7957-I7957

[12] Y. Zhang, F. Yang, and Y. X. Huang, "A novel SelectionLearning algorithm for multi-satellite scheduling problems", 2007 Ieee Congress on Evolutionary Computation, Vol. 1-10, 2007, pp. 1318-1324.

[13] E. Mok and P. A. Cross, "A Fast Satellite Selection Algorithm for Combined Gps and Glonass Receivers", Journal of Navigation, Vol. 47, Sep 1994, pp. 383-389.

[14] N. K. An-Lin Tao, and Shau-Shiun Jan, "Ionospheric Correction and Satellite Selection Techniques for the Medium-range Baseline RTK Positioning with GPS and QZSS", in International Technical Meeting 2015 (ITM) of Institute of Navigation, 2015.

[15] D. H. Won, J. Ahn, S.-W. Lee, J. Lee, S. Sung, and H.-W. Park, et al., "Weighted DOP With Consideration on Elevation-Dependent Range Errors of GNSS Satellites", IEEE Transactions on Instrumentation and Measurement, Vol. 61, Dec 2012, pp. 3241-3250 